\title{
Simulation and experimental results for the detection of conversion electrons with gas proportional scintillation counters ${ }^{\text {th }}$
}

\author{
P.J.B.M. Rachinhas ${ }^{a}, *$ P.C.P.S. Simões ${ }^{a}$, J.A.M. Lopes ${ }^{a}$, , T.H.V.T. Dias ${ }^{a}$, \\ R.E. Morgado ${ }^{c}$, J.M.F. dos Santos ${ }^{\mathrm{a}}$, A.D. Stauffer ${ }^{\mathrm{d}}$, C.A.N. Conde ${ }^{\mathrm{a}}$ \\ ${ }^{a}$ Departamento de Física, Universidade de Coimbra, 3004-516 Coimbra, Portugal \\ binstituto Superior de Engenharia de Coimbra, 3001-601 Coimbra, Portugal \\ ${ }^{\mathrm{c}}$ Los Alamos National Laboratory, Los Alamos, NM 87545, USA \\ ${ }^{\mathrm{d}}$ Department of Physics and Astronomy, York University, Toronto, Canada M3J 1P3
}

Received 24 May 1999; received in revised form 6 August 1999; accepted 26 August 1999

\begin{abstract}
The application of gas proportional scintillation counters (GPSC) to the detection and identification of conversion electrons in the medium energy range is investigated. Experimental and Monte Carlo results are presented for the response of a xenon GPSC, filled at atmospheric pressure, to the decay of a ${ }^{109} \mathrm{Cd}$ source. This source emits $88.0 \mathrm{keV}$ $\gamma$-rays, $\mathrm{e}_{\mathrm{L}}=84.6 \mathrm{keV}$ and $\mathrm{e}_{\mathrm{K}}=62.5 \mathrm{keV}$ conversion electrons, as well as fluorescence X-rays and Auger electrons. Good agreement is found between the measured and the calculated energy spectra. The response to higher-energy electrons is investigated by Monte Carlo simulation, by considering a hypothetical GPSC filled with xenon at 10 atm and doped with the ${ }^{133 \mathrm{~m}} \mathrm{Xe}$ metastable isotope. The calculated energy spectra for the absorption of the ${ }^{133 \mathrm{~m}} \mathrm{Xe} 233.2 \mathrm{keV} \gamma$-rays, $\mathrm{e}_{\mathrm{K}}=198.6 \mathrm{keV}$ and $\mathrm{e}_{\mathrm{L}}=228.4 \mathrm{keV}$ conversion electrons, as well as fluorescence X-rays and Auger electrons, are presented and discussed. (C) 2000 Elsevier Science B.V. All rights reserved.
\end{abstract}

Keywords: Gas scintillation detectors; Conversion electrons; Monte Carlo simulation; Digital pulse processing

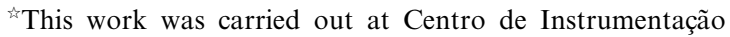
(Research Unit No. 217/94), Departamento de Física, Universidade de Coimbra, and received support from Junta Nacional de Investigação Científica e Tecnológica (JNICT), Lisboa, through Project CERN/P/FIS/1166/97, and from Los Alamos National Laboratory, Los Alamos, NM, USA, through Project F67630017-35/LANL/USA. The authors are grateful to Centro de Física Teórica for the use of computing facilities.

* Corresponding author. Tel.: + 351-39-410667; fax: + 35139-829158.

E-mail address: paulo@saturno.fis.uc.pt (P.J.B.M. Rachinhas)
}

\section{Introduction}

Xenon gas proportional scintillation counters (GPSC) are routinely used to detect X-rays when good energy resolution combined with high detection efficiency and room-temperature operation are required. This type of detector is used in many applications including astrophysics, medical instrumentation, X-ray fluorescence analysis and high-energy physics [1-6]. In this paper we in vestigate the application of GPSC technology to the analysis of the absorption and de-excitation 
processes that accompany the radioactive decay of metastable isotopes. The goal is to use GPSCs to detect and selectively identify conversion electrons (CE) in the medium energy range.

The GPSC associates a good energy resolution with the internal counting capability of gaseous detectors, allowing well-resolved conversion electron peaks. In addition, a GPSC can efficiently detect CEs in coincidence with the associated $\mathrm{K}$ fluorescence X-rays, allowing the identification of a CE event even in the presence of large backgrounds, particularly from $\beta$-decay.

Using both experimental measurements and Monte Carlo techniques, we analyse the response of a xenon GPSC to the radioactive decay of a ${ }^{109} \mathrm{Cd}$ source placed on the inside surface of the GPSC entrance window. Pulse signature analysis, including amplitude versus pulse time-duration analysis, was performed in the digital processing of the experimental data.

The response of a xenon GPSC to higher-energy conversion electrons is investigated by considering the decay of the ${ }^{133 \mathrm{~m}} \mathrm{Xe}$ metastable isotope. The metastable isotopes of xenon are signatures for all fission processes, including nuclear reactors, nuclear reprocessing, as well as nuclear detonations, and a determination of the ratios of the metastable isotopes can be used to determine their origin $[7,8]$. However, the detection of the high-energy conversion electrons from ${ }^{133 \mathrm{~m}} \mathrm{Xe}$ would require the fabrication of a high-pressure GPSC, and this was beyond the scope of our current experimental resources. Therefore, only a Monte Carlo simulation is used to model and predict the response of a $10 \mathrm{~atm}$ pressurized xenon GPSC doped with ${ }^{133 \mathrm{~m}} \mathrm{Xe}$ atoms.

The decay modes of ${ }^{109} \mathrm{Cd}$ and ${ }^{133 \mathrm{~m}} \mathrm{Xe}$ [9] are schematically represented in Figs. 1 and 2, respectively.

As shown in Fig. 1, ${ }^{109} \mathrm{Cd}$ decays by electron capture (EC) to ${ }^{109 \mathrm{~m}} \mathrm{Ag}$. The EC process occurs from $\mathrm{K}, \mathrm{L}$, and outer shells with probabilities $79 \%$, $17 \%$ and $4 \%$, respectively, but only $\mathrm{K}$-electron capture is represented. As indicated, the daughter element ${ }^{109} \mathrm{Ag}$ decays by the emission of $88.0 \mathrm{keV}$ $\gamma$-rays with a $4 \%$ probability, or by internal conversion (IC) with the probabilities $45 \%$ and $48 \%$ for the $\mathrm{K}$ and $\mathrm{L}$ shells. Internal conversion from shells higher than $\mathrm{L}$ contribute the remaining $3 \%$. The

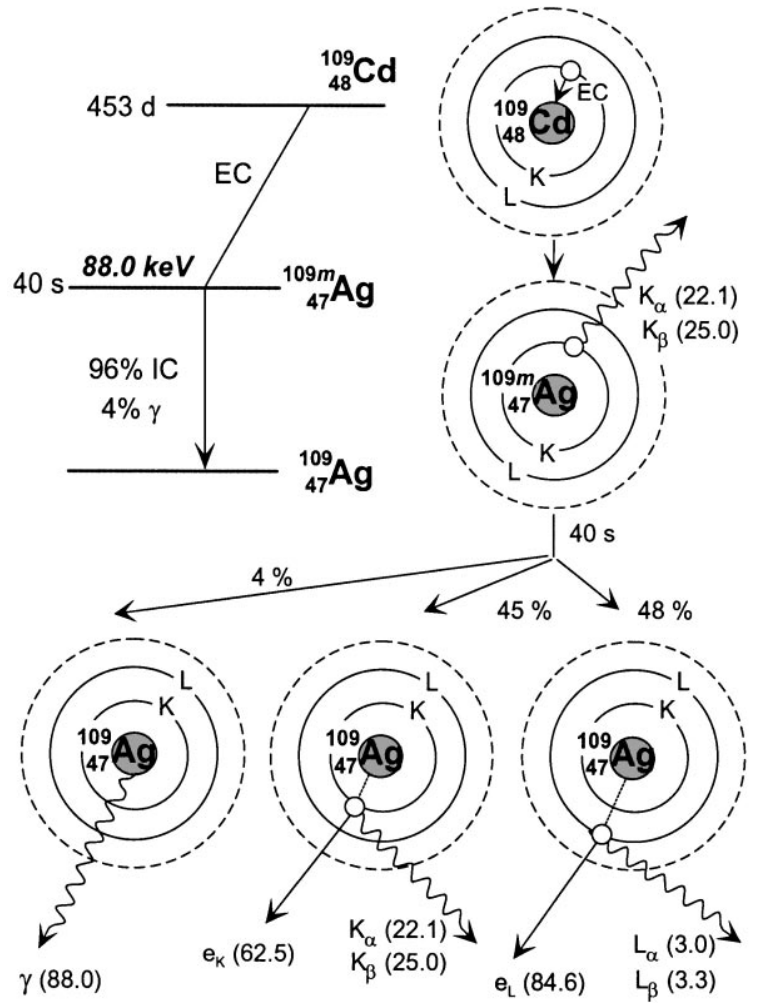

Fig. 1. Decay scheme of ${ }^{109} \mathrm{Cd}$ (numbers in parenthesis indicate energy values in $\mathrm{keV}$ ).

$\mathrm{K}$ and $\mathrm{L}$ IC channels involve the ejection of a conversion electron with energy $\mathrm{e}_{\mathrm{K}}=62.5 \mathrm{keV}$ or $\mathrm{e}_{\mathrm{L}}=84.6 \mathrm{keV}$, accompanied by the emission of a $\mathrm{Ag} \mathrm{K}$ - or L-fluorescence photon $\left(\mathrm{K}_{\alpha}=22.1\right.$, $\mathrm{K}_{\beta}=25.0 \mathrm{keV}$, or $\left.\mathrm{L}_{\alpha}=3.0, \mathrm{~L}_{\beta}=3.3 \mathrm{keV}\right)$ or by the emission of Auger electrons (not represented). Note that after electron capture, the emission of Ag fluorescence X-rays or Auger electrons also occurs.

Similarly, as shown in Fig. 2, the ${ }^{133 \mathrm{~m}} \mathrm{Xe}$ isotope decays by the emission of $233.2 \mathrm{keV} \gamma$-rays or by $\mathrm{K}$ or $\mathrm{L}$ internal conversion with the probabilities $10 \%, 67 \%$ and $23 \%$ as indicated. These channels add up to $100 \%$, since IC from shells higher than $\mathrm{L}$ give a negligible contribution. The IC channels involve the emission of a conversion electron with energy $\mathrm{e}_{\mathrm{K}}=198.6 \mathrm{keV}$ or $\mathrm{e}_{\mathrm{L}}=228.4 \mathrm{keV}$, accompanied by the emission of a K- or L-fluorescence $\mathrm{X}$-ray photon $\left(\mathrm{K}_{\alpha}=29.8 \mathrm{keV}, \mathrm{K}_{\beta}=33.8 \mathrm{keV}\right.$, or 


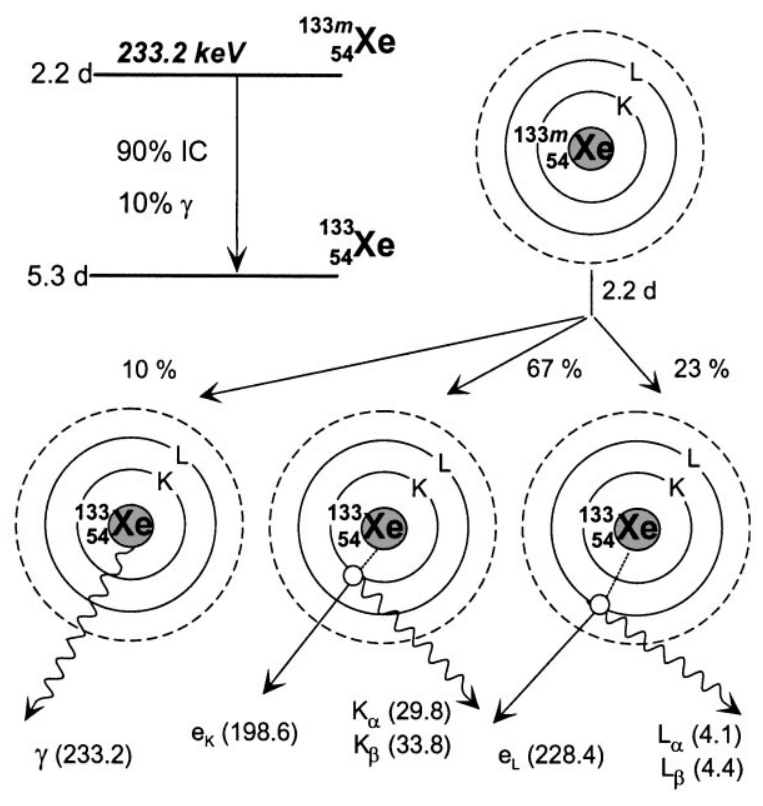

Fig. 2. Decay scheme of ${ }^{133 \mathrm{~m}} \mathrm{Xe}$ (numbers in parenthesis indicate energy values in $\mathrm{keV}$ ).

$\mathrm{L}_{\alpha}=4.1 \mathrm{keV}, \mathrm{L}_{\beta}=4.4 \mathrm{keV}$ ) or by the emission of Auger electrons (not represented).

\section{Results}

\subsection{Experimental results}

\subsubsection{Experimental setup}

The GPSC that was designed for measurements with the ${ }^{109} \mathrm{Cd}$ source is shown schematically in Fig. 3, and is similar to the detectors used in the previous work $[10,11]$.

It has a $10-\mathrm{cm}$ inner diameter body, a 4-cm thick absorption/drift region, and a 1-cm thick scintillation region. A 5.1-cm diameter quartz-window EMI D676QB photomultiplier tube (PMT) (a customized eight-dynode version of the 9266QB model) is used to detect the scintillation pulses. The non-magnetic stainless steel detector enclosure is topped by a 2.5 -cm diameter, $50 \mu \mathrm{m}$-thick Kapton $^{\text {TM }}$ radiation window, aluminized on the inner side to guarantee a uniform drift electric field in the absorption region. The first grid, G1 (made of

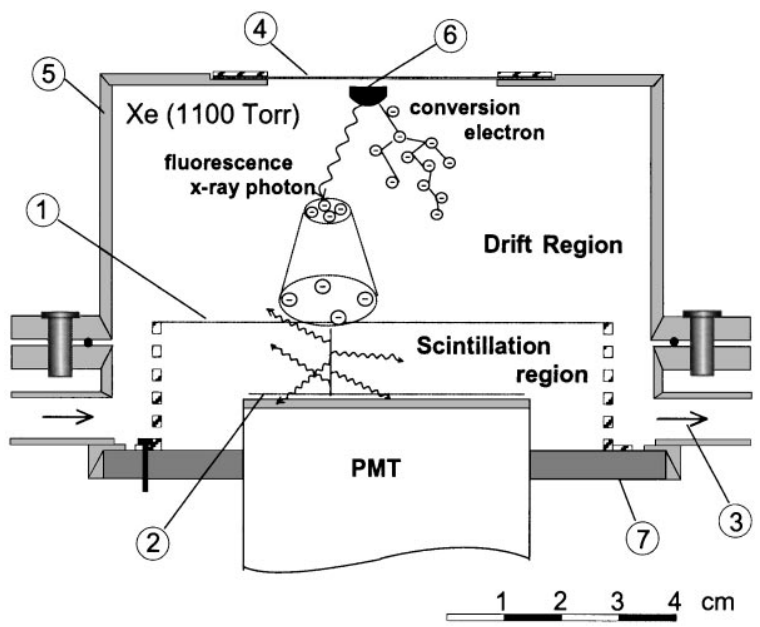

Fig. 3. Schematic of the gas proportional scintillation counter: (1) grid mesh with high electron transparency, G1; (2) grid G2 evaporated onto the photomultiplier (PMT); (3) to gas purifier; (4) Kapton ${ }^{\mathrm{TM}}$ window; (5) stainless-steel enclosure; (6) ${ }^{109} \mathrm{Cd}$ conversion electron source; (7) Macor ${ }^{\mathrm{TM}}$ insulator.

$80 \mu \mathrm{m}$ diameter stainless steel wire with a $900 \mu \mathrm{m}$ spacing) is supported by a multi-perforated stainless-steel cylinder, which is screwed to a Macor ${ }^{\mathrm{TM}}$ insulator base, one of the screws being used as a voltage feedthrough. The grid G2 is a $100 \mu \mathrm{m}$ line width and $1000 \mu \mathrm{m}$ spaced chromium grid, vacuum-evaporated onto the PMT window. The lateral surface of the PMT is coated with a vacuumdeposited chromium film, which acts also as a feedthrough to G2. Together with the G1-support cylinder, this film contributes to guarantee a well behaved uniform electric field at the edges of the scintillation region. A very low outgassing epoxy was used in the vacuum-tight assembly of the Macor ${ }^{\mathrm{TM}}$ base, the PMT and the radiation window. The fact that the scintillation region is in direct contact with the PMT window eliminates the need for an extra vacuum ultraviolet (VUV) window, maximizing the collection efficiency of the VUV scintillation by the PMT and reducing solid angle dependence [12,13].

The GPSC was filled with high-purity xenon (N45) at 1100 Torr through a SAES Model 150 gas purifier. The gas is continuously purified by SAES ST707/washer/833 getters, and is maintained in circulation by convection. Typical values for 
polarizing voltages are $V_{\mathrm{G} 1}=1500 \mathrm{~V}, \mathrm{~V}_{\mathrm{G} 2}=5500 \mathrm{~V}$ on the scintillation grids (yielding reduced electric fields of $\sim 0.3$ and $\sim 4 \mathrm{~V} \mathrm{~cm}^{-1} \mathrm{Torr}^{-1}$ in the drift and in the scintillation regions, respectively), and $\mathrm{V}_{\mathrm{k}}=5500 \mathrm{~V}, \mathrm{~V}_{\mathrm{a}}=6200 \mathrm{~V}$ on the PMT photocathode and anode.

The primary (sub-ionization) electron clouds produced by ionizing radiation in the absorption region of the GPSC drift under the influence of a low electric field into the scintillation region where, under higher fields, electroluminescence (VUV scintillation) is produced in xenon. The pulse resulting from the scintillation burst collected by the PMT is fed through a charge pre-amplifier and a main linear amplifier to a digital pulse-height analyzer (DPHA) developed by our group [14-16]. The main amplifier is operated with very short shaping times (50 ns), and as a result pulse shapes closely resemble the scintillation light burst. This produces an efficient pulse-shape discrimination. Each pulse is digitized by a hybrid ADC at a 20 $\mathrm{MHz}$ rate with 8-bit resolution before being processed by the DPHA.

In a typical run, each pulse is processed by a series of algorithms, as follows. To reduce highfrequency oscillation, the digitized pulse samples are smoothed by a three-point median filter. The pulse amplitude is obtained by numerical integration of the pulse samples. Pulse duration and pulse separation are taken as the intervals during which the signal is, respectively, above and below a threshold value, set just above noise level. Whenever a sampled value overshoots the ADC scale the pulse is rejected [16].

The detector performance characteristics were found to be similar to the other GPSCs made by our group. Its energy linearity and energy resolution, spatial uniformity, and detector response as a function of X-ray energy can be found in detail in several of our previous publications $[10,11]$.

As an example, we present in Fig. 4 the pulseheight distributions for three external and collimated X-ray/ $\gamma$-ray sources: ${ }^{55} \mathrm{Fe}$ (chromium filtered), ${ }^{109} \mathrm{Cd}$ and ${ }^{241} \mathrm{Am}$. The energy resolutions are about $8.5 \%, 4.9 \%$ and $4.2 \%$ for the $5.9,22.1$ and $59.6 \mathrm{keV} \mathrm{X}$-rays obtained from the three sources, respectively.
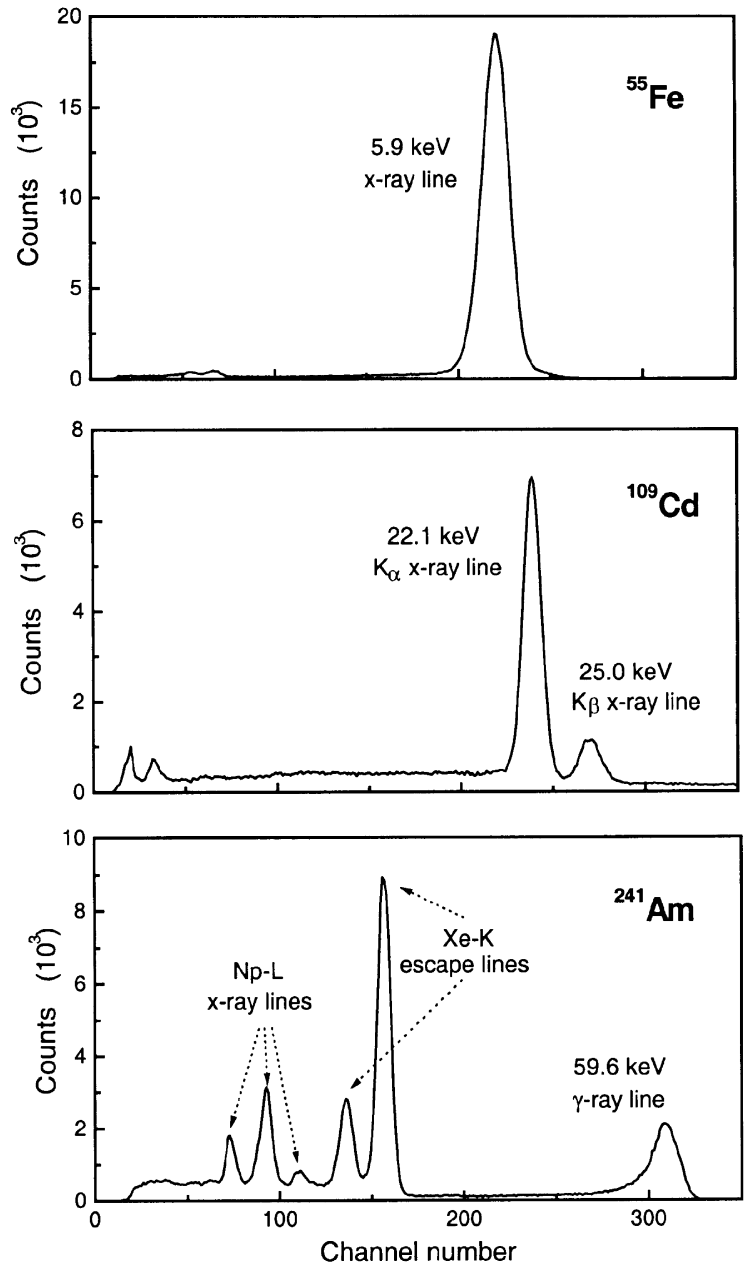

Fig. 4. Pulse-height distributions for the X-ray sources ${ }^{55} \mathrm{Fe},{ }^{109} \mathrm{Cd}$ and ${ }^{241} \mathrm{Am}$, measured with the present GPSC.

\subsubsection{Conversion electron measurements}

The GPSC experimental measurements were obtained with a non-collimated ${ }^{109} \mathrm{Cd}$ source placed within the active volume of the detector, at the top of the absorption/drift region, as shown in Fig. 3. The pressure of 1100 Torr was chosen in order to fully absorb the most energetic conversion electrons from the ${ }^{109} \mathrm{Cd}$ source in the $4-\mathrm{cm}$ deep active volume of the GPSC. At this pressure most $88.0 \mathrm{keV} \gamma$-rays escape from the detector.

Curve $a$ in Fig. 5 represents the raw pulse-height distribution that was obtained with the ${ }^{109} \mathrm{Cd}$ 


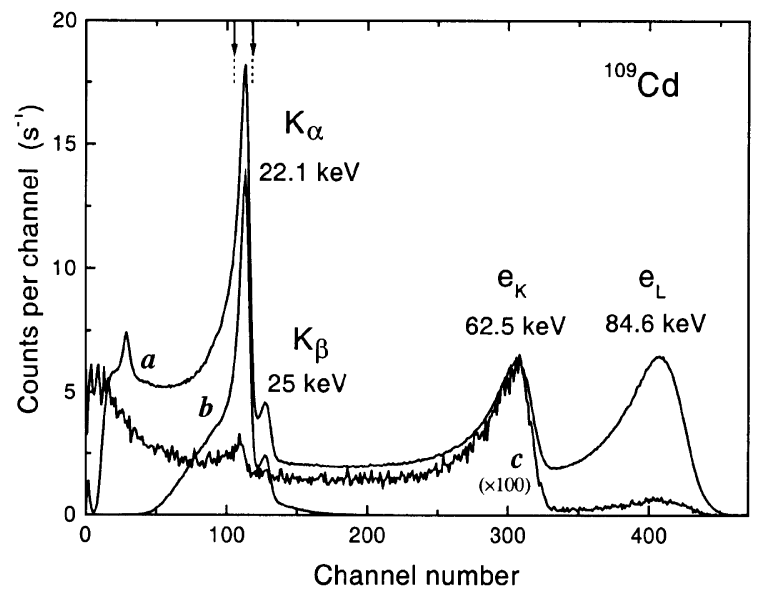

Fig. 5. Pulse-height distributions obtained from a non-collimated ${ }^{109} \mathrm{Cd}$ radioactive source placed inside the GPSC. Curve $a$ is the raw distribution. Curve $b$ is obtained when pulses with duration outside the range from 3.6 to $4.0 \mu$ s are rejected. Curve $c$ counts only the first pulse that appears within a $20 \mu \mathrm{s}$ interval after a $22.1 \mathrm{keV}$ trigger-pulse, which is chosen from the 3.6-4.0 $\mu$ s pulses that fall in the narrow range of the $\mathrm{K}_{\alpha}$-peak maximum between the channels indicated by the two arrows. Note that curve $c$ is multiplied by 100 .

source. The total count rate is about $2 \times 10^{3}$ counts per second. The $\mathrm{Ag} \mathrm{K} \mathrm{K}_{\alpha}=22.1 \mathrm{keV}$ and $\mathrm{K}_{\beta}=25.0 \mathrm{keV}$ fluorescence lines and the $\mathrm{e}_{\mathrm{K}}=62.5 \mathrm{keV}$ and $\mathrm{e}_{\mathrm{L}}=84.6 \mathrm{keV}$ CE peaks can be distinguished. The low-energy tail associated with each peak results from solid-angle effects in the collection of the VUV scintillation photons, from the angle dependent reflection of the VUV photons by the PMT quartz window, and from loss of electrons to the detector walls since the radioactive source is not collimated. In addition, a fraction of the Auger electrons resulting from $\mathrm{Ag} \mathrm{K}$-vacancy decay (average energy $19.7 \mathrm{keV}$ [17]) may leave the source volume with an energy distribution due to straggling inside the source, and interact in the xenon gas. These electrons will give a contribution to the tail of the $\mathrm{K}_{\alpha}$-peak, as well as to the $\mathrm{e}_{\mathrm{L}}$-peak tail when combined with the $\mathrm{e}_{\mathrm{K}}$ electrons.

If we consider that each peak of the pulse-height distribution is composed of a low-energy tail superimposed on a Gaussian peak, the energy resolutions of the $\mathrm{K}_{\alpha}, \mathrm{e}_{\mathrm{K}}$ and $\mathrm{e}_{\mathrm{L}}$ peaks are $9 \%, 12 \%$, and $11 \%$, respectively. The peak areas are clearly not in accordance with the relative intensities of the decay channels for the $\mathrm{K}_{\alpha}, \mathrm{e}_{\mathrm{K}}$ and $\mathrm{e}_{\mathrm{L}}$ events, which is $84: 45: 48$ (see Fig. 1). ${ }^{1}$ This is because at 1100 Torr about $50 \%$ of the $\mathrm{K}_{\alpha}$ photons escape from the detector, and because, in addition, combined $\mathrm{K}_{\alpha}$ and $\mathrm{e}_{\mathrm{K}}$ events may contribute to the $\mathrm{e}_{\mathrm{L}}$ peak. In fact, the primary electron clouds produced by a fraction $(\sim 12 \%)$ of the $K_{\alpha}$ events reach the scintillation region simultaneously with an $\mathrm{e}_{\mathrm{K}}$ cloud, a situation where an $\mathrm{e}_{\mathrm{K}}$ event becomes indistinguishable from an $\mathrm{e}_{\mathrm{L}}$ event.

Using pulse-duration discrimination, the background under the $22.1 \mathrm{keV}$ X-ray peak can be efficiently reduced, as shown by curve $b$ in Fig. 5, which represents the pulse-height distribution obtained when only the 3.6 to $4.0 \mu$ s pulses are counted.

The implementation of a gated-GPSC technique $[19,20]$ permits a selective identification of the $e_{K}$ conversion electron events, making use of the fact that a large fraction of the $\mathrm{e}_{\mathrm{K}}$ events are associated with the emission of a $K_{\alpha}$ photon $(68 \%)$, and that a unique pulse signature is produced whenever the electron clouds from an $\mathrm{e}_{\mathrm{K}}$ electron and a $\mathrm{K}_{\alpha}$ fluorescence $\mathrm{X}$-ray reach the scintillation region separately.

In Fig. 5, curve $c$ represents the pulse-height distribution where every pulse counted is the first pulse that appears within a $20 \mu$ s window after a trigger-pulse. From the pulses with a 3.6 to $4.0 \mu \mathrm{s}$ duration (included under curve $b$ ), the pulses selected for triggering are those falling in the narrow range of the $\mathrm{K}_{\alpha}$-peak maximum between the channels indicated by the two arrows in Fig. 5. Examples of trigger-pulse/counted-pulse pairs are depicted in Fig. 6.

The gating improved considerably the selectivity of the system to the $\mathrm{e}_{\mathrm{K}}$ events as shown in curve $c$ of Fig. 5, where the $e_{K}$ peak appears as the only prominent feature. When compared with the raw pulse-height distribution represented by curve $a$,

\footnotetext{
${ }^{1} \mathrm{~A}$ K-vacancy in Ag decays by $\mathrm{K}_{\alpha}, \mathrm{K}_{\beta}$ or Auger emission with probabilities $68 \%, 15 \%$ and $17 \%$, respectively [18]. The value 84 is obtained for $\mathrm{K}_{\alpha}$ when we take into account that $\mathrm{K}_{\alpha}$ photons are produced both in the ${ }^{109} \mathrm{Cd} \mathrm{EC}(\mathrm{K})$ and $\mathrm{IC}(\mathrm{K})$ channels $(0.79 \times 0.68+0.45 \times 0.68=0.84)$.
} 


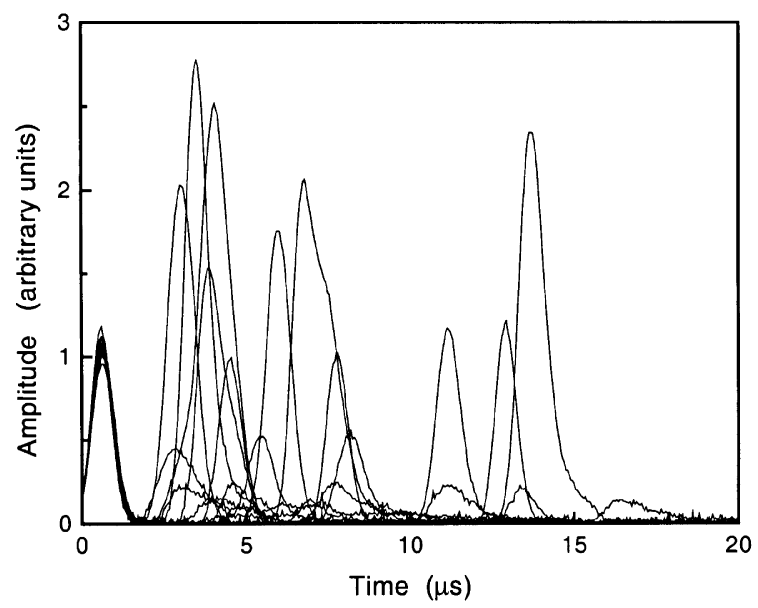

Fig. 6. Set of digitized pulses obtained with a ${ }^{109} \mathrm{Cd}$ radioactive source placed inside the GPSC. Each curve corresponds to a decay event, showing the initial ${ }^{109} \mathrm{Ag} \mathrm{K}_{\alpha}$-fluorescence trigger-pulse followed by a second pulse within a $20 \mu$ s window.

the energy resolution is slightly improved, from $12 \%$ to $10 \%$, but the peak-to-background ratio exhibits a significant increase from 2 to 6 . However, the detection efficiency is reduced by about two orders of magnitude in curve $c$, since the method is limited by the $\mathrm{K}_{\alpha}$-yield, by the probability that the $\mathrm{K}_{\alpha}$ photon and $\mathrm{e}_{\mathrm{K}}$ electron pair are both emitted in the forward hemisphere, by the detector efficiency for the absorption of the $\mathrm{K}_{\alpha} \mathrm{X}$-rays, and by the fact that many $K_{\alpha}$ interactions do not result in full amplitude pulses, and are thus not selected for triggering.

\subsection{Monte Carlo results}

In this section we describe results of Monte Carlo simulations of the response of a model xenon GPSC to the decay emissions of the radioactive ${ }^{109} \mathrm{Cd}$ and ${ }^{133 \mathrm{~m}} \mathrm{Xe}$ isotopes.

In Section 2.2.1 a brief description of the Monte Carlo simulation is given, including a summary of the cross-section data used in the calculations.

In Section 2.2.2, we present the results of a Monte Carlo simulation of the response of a 1100 Torr xenon GPSC detector to the $\mathrm{e}_{\mathrm{K}}=62.5 \mathrm{keV}$ and $\mathrm{e}_{\mathrm{L}}=84.6 \mathrm{keV}$ conversion electrons and to the $\mathrm{K}_{\alpha}=22.1 \mathrm{keV}$ and $\mathrm{K}_{\beta}=25.0 \mathrm{keV}$
$\mathrm{X}$-rays from a ${ }^{109} \mathrm{Cd}$ source and compare them with the experimental measurements. The $88.0 \mathrm{keV}$ ${ }^{109} \mathrm{Cd} \gamma$-rays are neglected, since their contribution to the spectrum is very small (the $\gamma$-ray decay channel contributes $4 \%$ and only $10 \%$ of the $\gamma$-rays interact inside the detector). As well, the emissions of L-fluorescence X-rays and all Auger electrons are not included, although they may not be totally absorbed inside the source.

In Section 2.2.3, we consider a xenon GPSC detector pressurized to 10 atmospheres and doped with ${ }^{133 m} \mathrm{Xe}$. We present results for the energy spectrum corresponding to the interaction with the xenon filling of the ${ }^{133 \mathrm{~m}} \mathrm{Xe} 233.2 \mathrm{keV} \gamma$-rays, $\mathrm{e}_{\mathrm{K}}=198.6 \mathrm{keV}$ and $\mathrm{e}_{\mathrm{L}}=228.4 \mathrm{keV}$ conversion electrons, and all fluorescence, Auger and shake-off electron emissions.

\subsubsection{Description}

The emission of $\gamma$-rays and conversion electrons by the ${ }^{109} \mathrm{Cd}$ source or by the ${ }^{133 \mathrm{~m}} \mathrm{Xe}$ atoms is assumed to be isotropic. Isotropy is also assumed for the emission of fluorescence X-rays and $\mathrm{Au}$ ger/Coster-Kronig or shake-off electrons.

The Monte Carlo simulation includes the electron impact ionization of the Xe atoms, the photoionization of the Xe atoms by the $\gamma$-rays and X-rays as well as the complex vacancy cascade decay of the residual $\mathrm{Xe}$ ions to multi-charged ionic ground states. This process involves the emission of $\mathrm{Xe}$ Auger/Coster-Kronig and shake-off electrons as well as Xe fluorescence X-rays. All electrons are followed individually through their multiple elastic and inelastic (excitation and ionization) collisions in Xe, down to sub-ionization energies, i.e., until the primary electron cloud is fully developed. The production of bremsstrahlung was neglected, since for the electron energies involved, radiative losses represent less than $2 \%$ of the total energy degradation [21,22].

Once the primary electron cloud is formed, the time and position characteristics of the electrons in the cloud are determined from the diffusion equation, up to the point where they reach the first grid (G1) of the detector. Diffusion is neglected after the electrons enter the higher field scintillation region, i.e. electrons are assumed to follow a straight path from grid G1 towards grid G2. It is also assumed 
that the VUV scintillation photons produced while electrons drift along this region are emitted isotropically. For each value of the applied reduced electric field, the adopted rate of emission is the scintillation yield obtained in previous Monte Carlo studies $[23,24]$. The calculations have taken into account the quartz-window transmission as a function of the VUV photon incidence angle.

For the cross-section data used in the Monte Carlo calculations see also Refs. [23,24].

The total and partial photoionization crosssection used to simulate the absorption of $\gamma$-rays and $\mathrm{X}$-rays in xenon are taken from the data in Refs. [25-30]. To take into account the direction in which the photoelectron is emitted, we use the data from [27].

The vacancy cascade decay of the residual Xe ions after photoionization or electron impact ionization is reproduced using the Auger/Coster-Kronig transition rates from Refs. [31-34] and the fluorescence transition rates from Refs. [35,36]. The emission of a shake-off electron after either a photoionization event or an Auger/Coster-Kronig electron emission is determined by the probabilities described in Ref. [37]. In every case, rates are adjusted when necessary for transitions from less than a full shell which occur during the cascade decay.

For elastic collisions of electrons in xenon, the low-energy integral and differential cross-sections adopted are described in Refs. [38-40]. Above 20 $\mathrm{eV}$, integral elastic cross-section data are taken from Ref. [41]. Elastic angular differential crosssections above $20 \mathrm{eV}$ value, are based on Ref. [42] up to $3 \mathrm{keV}$ and on Ref. [43] for higher energies.

The partial excitation cross-sections for 12 xenon excited states are taken from the data of Ref. [44], and their sum is taken as the total excitation crosssection. For the angular differential cross-sections, a simple linear representation as a function of scattering angle $\theta$ is used, where the slopes are a quadratic function of the electron energy.

The total ionization cross-section is based on the experimental results of Refs. [45,46]. Electron impact inner-shell ionization is taken into account, and we use the partial ionization cross-sections of Ref. [47]. To calculate the sharing of the excess electron energy between the two outgoing

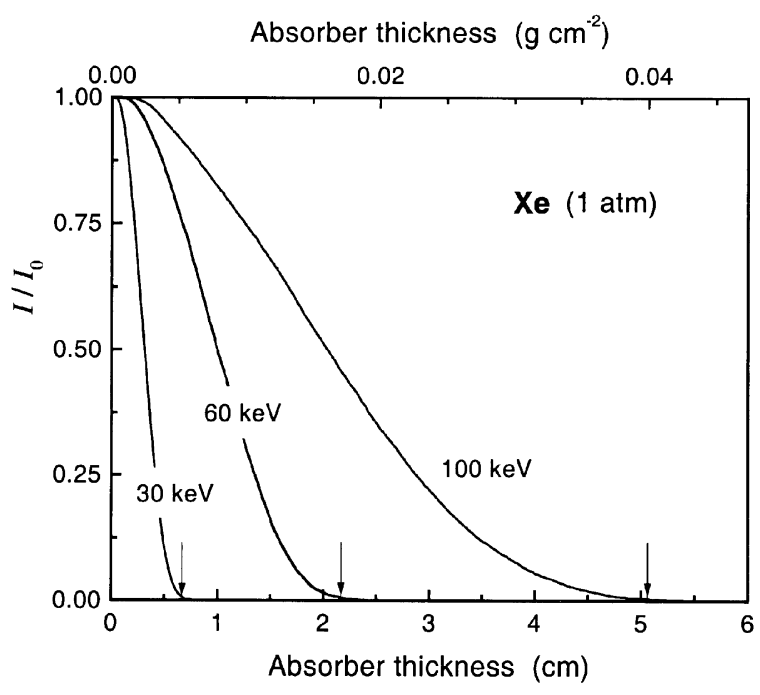

Fig. 7. Calculated transmission curves for electrons in xenon at atmospheric pressure for three electron energies. The arrows indicate the electron ranges taken from [22].

electrons, we used the shape of the energy differential cross-sections described by Ref. [48] for He, with the help of their measurements in xenon for the unique energy of $500 \mathrm{eV}$. For the angular scattering, we assume that the primary (fast) electron is always scattered forward, and the secondary is ejected at right angles.

For the electron energies relevant to the present work, we tested the Monte Carlo calculated electron ranges against data in the literature $[22,49]$ and the choice of cross-sections was refined accordingly. As an example, the transmission curves calculated by the present Monte Carlo simulation for 30, 60 and $100 \mathrm{keV}$ electrons are presented in Fig. 7, showing a very good agreement of the corresponding electron ranges with values from Ref. [22] for the same energies.

\subsection{2. ${ }^{109} \mathrm{Cd}$ results}

The GPSC geometry adopted for the calculations matches the conditions of the GPSC experimental set-up, as shown in Fig. 8. However, the electric fields applied in the drift and scintillation regions are assumed to be uniform in the entire volumes. A pressure of 1100 Torr was considered for the xenon filling. 


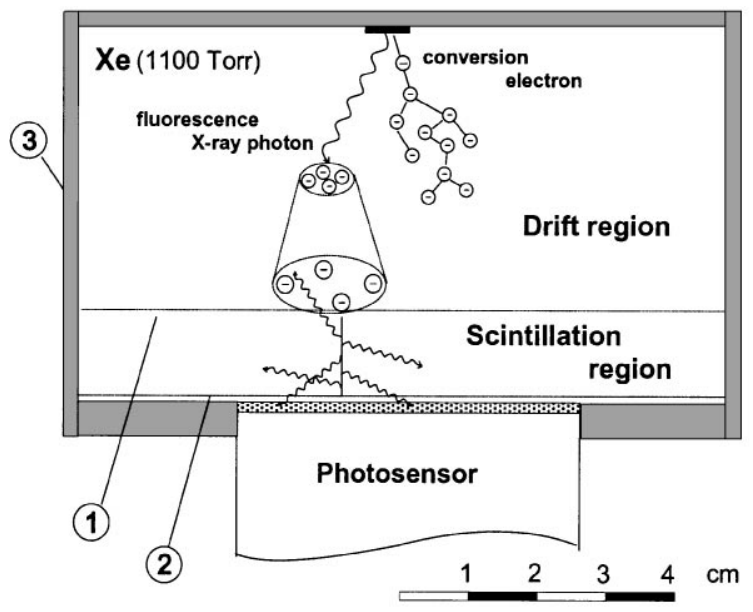

Fig. 8. Detector geometry adopted for the Monte Carlo simulations: (1) first grid, G1; (2) second grid, G2; (3) enclosure.

Fig. 9 shows the Monte Carlo energy spectrum calculated for the absorption of the $\mathrm{e}_{\mathrm{K}}$ and $\mathrm{e}_{\mathrm{L}}$ conversion electrons and the $\mathrm{K}_{\alpha}$ and $\mathrm{K}_{\beta} \mathrm{X}$-rays from the hypothetical ${ }^{109} \mathrm{Cd}$ source in the GPSC. The tail of the $e_{L}$ peak is higher than the tail of the $\mathrm{e}_{\mathrm{K}}$ peak because $\mathrm{e}_{\mathrm{L}}$ electrons have a longer range in the gas so that losses to the walls are more important. Also, the observed broadening of the $84.6 \mathrm{keV}$ peak is the result of the summing of several different conversion electron and fluorescence decay products.

The calculated spectrum in Fig. 9 can be compared with the experimental pulse-height distribution, curve $a$ in Fig. 5. We observe that the experimental spectrum peaks are broader and exhibit more pronounced low-energy tails than the calculated spectrum. This can be attributed to the energy loss of the CE and Auger electrons inside the source, and to the non-uniformity of the electric field near the scintillation region boundaries, two effects that were not included in the simulation.

The energy resolutions $R$ of the 22.1, 62.5 and $84.6 \mathrm{keV}$ peaks in the calculated spectrum in Fig. 9 are $6 \%, 5 \%$ and $8 \%$, respectively. These values are lower than the corresponding $R$ results for the experimental spectrum $(9 \%, 12 \%$ and $11 \%)$, reflecting the higher peak tails in the measured

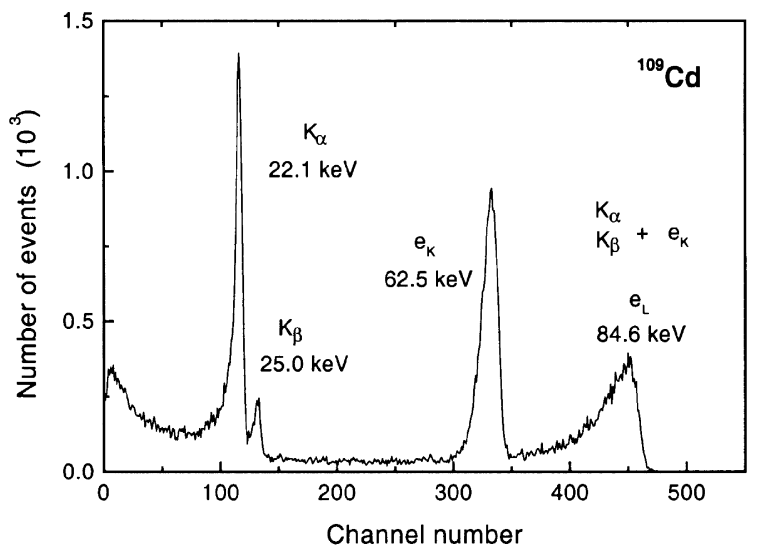

Fig. 9. Monte Carlo energy spectrum for the absorption of the $\mathrm{e}_{\mathrm{K}}=62.5 \mathrm{keV}$ and $\mathrm{e}_{\mathrm{L}}=84.6 \mathrm{keV}$ conversion electrons, as well as the 22.1 and $25.0 \mathrm{keV} \mathrm{K}_{\alpha}$ and $\mathrm{K}_{\beta}$-fluorescence $\mathrm{X}$-rays from the decay of a ${ }^{109} \mathrm{Cd}$ source.

distribution. For both the calculated and the experimental $R$ results, the variation from the first to the second $R$ value is slower than $1 / \sqrt{E}$. This effect, which is known to occur for increasing energy of ionizing radiation, is under investigation, and was shown to depend on the intensity of the electric field applied in the absorption region of the detector [50].

\subsection{3. ${ }^{133 \mathrm{~m}} \mathrm{Xe}$ results}

In this section we present Monte Carlo results obtained for a hypothetical GPSC detector with a 10 atm pressurized xenon filling doped with ${ }^{133 \mathrm{~m}} \mathrm{Xe}$ atoms. The detector geometry is similar to Fig. 8, but the absorption and scintillation regions are now 20.0 and $0.2 \mathrm{~cm}$ deep, respectively. The simulation includes the absorption of the $233.2 \mathrm{keV}$ $\gamma$-rays plus the $\mathrm{e}_{\mathrm{K}}=198.6 \mathrm{keV}$ and $\mathrm{e}_{\mathrm{L}}=228.4 \mathrm{keV}$ conversion electrons, taking into account the $10 \%$, $67 \%$ and $23 \%$ probabilities of the respective ${ }^{133 \mathrm{~m}} \mathrm{Xe}$ decay channels, as shown in Fig. 2. The position of the ${ }^{133 \mathrm{~m}} \mathrm{Xe}$ atoms is randomly chosen within the detector volume. For the chosen pressure the $\mathrm{e}_{\mathrm{L}}$ electron range is $\approx 2 \mathrm{~cm}$, and this guarantees that a minimum of $90 \%$ of the $\mathrm{e}_{\mathrm{L}}$ electrons are totally absorbed within the detector volume.

For the same number of decay events in each distribution, Fig. 10a represents the partial energy 

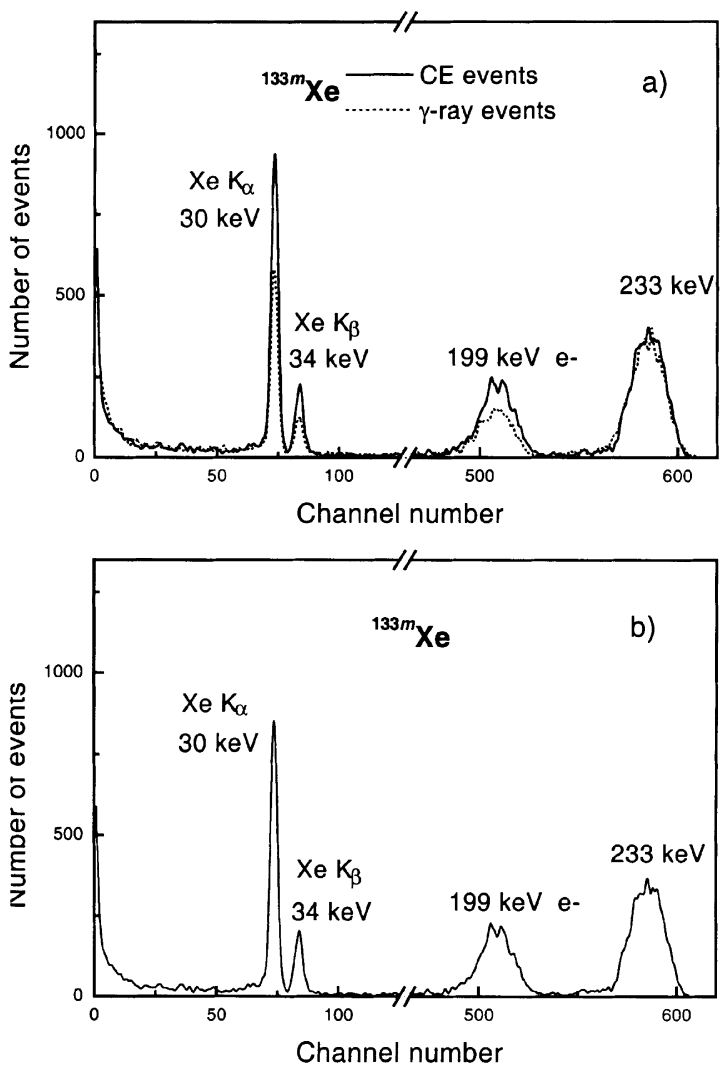

Fig. 10. Monte Carlo energy spectra for a 10 atm xenon GPSC doped with ${ }^{133 \mathrm{~m}} \mathrm{Xe}$ atoms. (a) Partial energy spectra counting $\gamma$-ray events $(\cdots)$ and conversion electron events $(-)$. (b) Total energy spectrum.

spectra obtained when only the $\mathrm{e}_{\mathrm{K}}$ and $\mathrm{e}_{\mathrm{L}}$ events (in the proportion 67:23) are absorbed or when only the $\gamma$-ray events are absorbed. The total energy spectrum is shown in Fig. 10b corresponding to $\gamma$-ray, $e_{K}$, and $e_{L}$ events in the proportion of $10 \%$ to $67 \%$ to $23 \%$. This spectrum is lower than the weighted sum of the two distributions from Fig. $10 \mathrm{a}$, since the effective contribution of $\gamma$-ray events is only $1 \%$ (only $10 \%$ of the $\gamma$-ray events interact within the detector volume).

In the partial and total distributions in Fig. 10, the peak at higher energy counts full-energy $(233.2 \mathrm{keV})$ absorption events. These are either ${ }^{133 \mathrm{~m}} \mathrm{Xe} \gamma$-ray events or ${ }^{133 \mathrm{~m}} \mathrm{Xe}$ internal conversion events where the corresponding photoelectron or conversion electron as well as all the Auger elec- trons and fluorescence X-rays resulting from the decay of the initial vacancy in the Xe residual ions are all absorbed in the xenon gas and detected as a single pulse.

However, for full-energy K-vacancy events (resulting from $\mathrm{K}$-shell photoionization by $\gamma$-rays or from $\mathrm{K}$ internal conversion) where the $\mathrm{K}$-vacancy decays by $\mathrm{K}_{\alpha}$ - or $\mathrm{K}_{\beta}$-fluorescence emission, the primary electron cloud produced by the $198.6 \mathrm{keV}$ electron and the cloud produced by the K-fluorescence photon may arrive separately at grid G1. In these circumstances, two distinct pulses are detected per event: one is counted in the intermediate peak and the other is counted under either of the well-defined $\mathrm{K}_{\alpha}$ or $\mathrm{K}_{\beta}$ peaks at lower energies. Note that the area of the intermediate peak is slightly higher than the area of the $\mathrm{K}_{\alpha}$ and $\mathrm{K}_{\beta}$ together, because the intermediate peak includes in addition the small number of events where the K-fluorescence X-rays escape from the detector.

On the other hand, we observe that the two partial energy spectra in Fig. 10a are very similar; only the areas of the individual peaks differ. These areas reflect the different probabilities for photoionization and internal conversion, as well as the geometry of emission (randomly positioned ${ }^{133 \mathrm{~m}} \mathrm{Xe}$ atoms). Although those probabilities are not the same, this does not affect the position of the peaks.

The similarity of the two partial distributions shows that the $\gamma$-ray events cannot be distinguished from the internal conversion events in terms of energy. In fact, because the metastable isotope and the detector medium are both xenon, the decay products of ${ }^{133 \mathrm{~m}} \mathrm{Xe}$ internal conversion for a given xenon-isotope shell (conversion electron and ${ }^{133 \mathrm{~m}} \mathrm{Xe}$ residual ion) are identical to the products of the interaction of the ${ }^{133 \mathrm{~m}} \mathrm{Xe} \gamma$-rays in the xenon medium for the same xenon shell (photoelectron and Xe residual ion). The same obviously applies for background $\gamma$-rays of similar energy. In these circumstances, the distinction will only become possible if a noble gas other than xenon, such as krypton or argon, is used for the detector filling, since this will in principle differentiate the $\gamma$-ray absorption products (associated with $\mathrm{Kr}$ or $\mathrm{Ar}$ photoionization) from the ${ }^{133 \mathrm{~m}} \mathrm{Xe}$ decay products. 


\section{Conclusions}

Experimental and Monte Carlo results have shown that it is possible to use GPSC techniques for the identification of energetic electrons, such as the internal conversion electrons (CE) produced in the decay of the ${ }^{109} \mathrm{Cd}$ and ${ }^{133 \mathrm{~m}} \mathrm{Xe}$ radioisotopes.

The GPSC associates a good energy resolution with the internal counting capability of gaseous detectors, allowing well-resolved conversion electron peaks and a selective identification of these electrons by making coincidences with the associated $\mathrm{K}$-fluorescence $\mathrm{X}$-rays.

The application of a gating technique to the experimental measurements obtained with a xenon GPSC with a ${ }^{109} \mathrm{Cd}$ allowed a selective identification of the $\mathrm{e}_{\mathrm{K}}=62.5 \mathrm{keV}$ conversion electrons from the daughter ${ }^{109} \mathrm{Ag}$. The $\mathrm{K}_{\alpha} \mathrm{X}$-rays associated with these $e_{K}$ events were used as a trigger.

For the case of the ${ }^{133 \mathrm{~m}} \mathrm{Xe}$ isotope, this gating technique would not be efficient, since the $K_{\alpha}$ fluorescence X-rays associated with $\mathrm{e}_{\mathrm{K}}$ conversion electron events are also associated with $\gamma$-ray events. In fact, because the metastable isotope and the GPSC filling are both xenon, the ${ }^{133 \mathrm{~m}} \mathrm{Xe}$ conversion electron events cannot be distinguished from the ${ }^{133 \mathrm{~m}} \mathrm{Xe} \gamma$-ray events, or from background radiation of similar energy. The distinction becomes in principle possible, though, if a noble gas other than xenon, such as krypton or argon, is chosen for the detector filling.

\section{References}

[1] C.A.N. Conde, L.R. Ferreira, M.F.A. Ferreira, IEEE Trans. Nucl. Sci. NS- 24 (1977) 221.

[2] A.J.P.L. Policarpo, Space Sci. Instr. 3 (1977) 77.

[3] A. Peacock, B.G. Taylor, N. White, T. Courvoisier, G. Manzo, IEEE Trans. Nucl. Sci. NS- 32 (1985) 108.

[4] V.P. Varvaritsa, I.K. Vikulov, V.V. Ivashov, M.A. Panov, V.I. Filatov, K.I. Shchekin, Instr. Exp. Tech. 35 (1992) 745.

[5] T.H.V.T. Dias, Physics of noble gas X-ray detectors: a Monte Carlo simulation study, in: Linking the Gaseous and Condensed Phases of Matter: The Behaviour of Slow Electrons, Nato Asi Series B: Physics, Vol. 326, Plenum Press, New York, 1994, pp. 543-559.

[6] T.H.V.T. Dias, J.M.F. dos Santos, P.J.B.M. Rachinhas, F.P. Santos, C.A.N. Conde, A.D. Stauffer, J. Appl. Phys. 82 (1997) 2742.
[7] W.R. Schell, M.J. Tobin, D.J. Marsan, C.W. Schell, J. Vives-Batlle, S.R. Yoon, Nucl. Instr. and Meth. A385 (1997) 277.

[8] G. Lamaze, Nucl. Instr. and Meth. A385 (1997) 285.

[9] C. Michael Lederer, V.S. Shirley (Eds.), Table of Isotopes, 7th Edition. Wiley, New York, 1978.

[10] J.M.F. dos Santos, A.C.S.M. Bento, C.A.N. Conde, X-ray Spectrom. 22 (1993) 328.

[11] J.M.F. dos Santos, J.F.C.A. Veloso, R.E. Morgado, C.A.N. Conde, Nucl. Instr. and Meth. A 353 (1994) 195.

[12] J.M.F. dos Santos, T.H.V.T. Dias, S.D.A.R. Cortes, C.A.N. Conde, Nucl. Instr. and Meth. A 280 (1989) 288.

[13] J.M.F. dos Santos, A.C.S.M. Bento, C.A.N. Conde, IEEE Trans. Nucl. Sci. NS-39 (1992) 541.

[14] P.C.P.S. Simões, J.C. Martins, C.M.B.A. Correia, IEEE Trans. Nucl. Sci. NS-43 (1996) 1804.

[15] P.C.P.S. Simões, J.M.F. dos Santos, C.A.N. Conde, IEEE Trans. Nucl. Sci. NS-45 (1998) 290.

[16] P.C.P.S. Simões, J.M.F. dos Santos, C.A.N. Conde, Nucl. Instr. and Meth. A 422 (1999) 341.

[17] T. Perkins, D.E. Cullen, M.H. Chen, J. Rathkopf, J. Scofield, Tables and graphs of atomic shell and relaxation data derived from the LLNL Evaluated Atomic Data Library (EADL), $Z=1-100$, Technical Report 30, Lawrence Livermore National Laboratory, 1991.

[18] R. Tertian, F. Claisse, Principles of Quantitative X-ray Fluorescence Analysis, Heyden, London, 1982.

[19] G. Manzo, J. Davelaar, A. Peacock, R.D. Andresen, B.G. Taylor, Nucl. Instr. and Meth. 177 (1980) 595.

[20] M.R. Sims, G. Manzo, A. Peacock, B.G. Taylor, Nucl. Instr. and Meth. 211 (1983) 499.

[21] G.F. Knoll, Radiation Detection and Measurements, 2nd Edition. Wiley, New York, 1989.

[22] L. Pages, E. Bertel, H. Joffre, L. Sklavenitis, Atom. Data 4 (1972) 1.

[23] T.H.V.T. Dias, F.P. Santos, A.D. Stauffer, C.A.N. Conde, Phys. Rev. A48 (1993) 2887.

[24] F.P. Santos, T.H.V.T. Dias, A.D. Stauffer, C.A.N. Conde, J. Phys. D 27 (1994) 42.

[25] M. Kutzner, V. Radojevic, H.P. Helly, Phys. Rev. A 40 (1989) 5052.

[26] I.M. Band, Yu.I. Kharitonov, M.B. Trzhaskovskaya, Atom. Data Nucl. Data Tables 23 (1979) 443.

[27] D.J. Kennedy, S.T. Manson, Phys. Rev. A 5 (1972) 227.

[28] F. Wuilleumier, Phys. Rev. A 6 (1972) 2067.

[29] E.B. Saloman, J.H. Hubbell, J.H. Scotfield, Atom. Data Nucl. Data Tables 38 (1988) 1.

[30] W.M. Veigele, Atom Data Nucl. Data Tables 5 (1973) 51.

[31] E.J. McGuire, M-shell Auger, Coster-Kronig and radiative matrix elements, and Auger and Coster-Kronig transition rates in $j-j$ coupling, Technical Report SC-RR71 0835, Sandia National Laboratories, 1972.

[32] E.J. McGuire, Phys. Rev. A 5 (1972) 1052.

[33] M.H. Chen, B. Crasemann, H. Mark, Atom. Data Nucl. Data Tables 24 (1979) 13. 
[34] E.J. McGuire, N-shell Auger, Coster-Kronig and radiative matrix elements, and Auger and Coster-Kronig transition rates in $j-j$ coupling. Technical Report SAND-75-0443, Sandia National Laboratories, 1975.

[35] J.H. Scofield, Atom. Data Nucl. Data Tables 14 (1974) 121.

[36] S.T. Manson, D.J. Kennedy, Atom. Data Nucl. Data Tables 14 (1974) 111.

[37] T.A. Carlson, C.W. Nestor, Phys. Rev. A 8 (1973) 2887.

[38] R.P. McEachran, A.D. Stauffer, J. Phys. B 19 (1986) 3523.

[39] A.D. Stauffer, T.H.V.T. Dias, C.A.N. Conde, Nucl. Instr. and Meth. A (1986) 242.

[40] R.P. McEachran, A.D. Stauffer, J. Phys. B 20 (1987) 3483.

[41] M. Hayashi, Recommended values of transport cross sections for elastic collisions and total collision cross sections for electrons in atomic and molecular gases, Technical Report IPPJ-AM-19, Nagoya University, 1981.

[42] I.E. McCarthy, C.J. Noble, B.A. Phillips, A.D. Turnbull, Phys. Rev. A 15 (1977) 2173.

[43] M. Fink, J. Kessler, Z. Phys. 196 (1966) 1.
[44] V. Puech, S. Mizzi, J. Phys. D 24 (1991) 1974.

[45] E. Khrishnakumar, K. Srivastava, J. Phys. B 21 (1988) 1055.

[46] B.L. Schram, Physica 32 (1966) 197.

[47] H. Deutsch, T.D. Mark, Contr. Plasma Phys. 34I (1994) 19.

[48] C.B. Opal, E.C. Beaty, W.K. Peterson, Tables of energy and angular distributions of electrons ejected from simple gases by electron impact, Technical Report 108, JILA, 1971.

[49] M.J. Berger, Nuclear and atomic data for radiotherapy and related radiobiology. Technical Report STI/PUB/741, IAEA, Vienna, 1987.

[50] P.J.B.M. Rachinhas, T.H.V.T. Dias, F.P. Santos, C.A.N. Conde, A.D. Stauffer, Absorption of electrons in xenon for energies up to $200 \mathrm{keV}$ : a Monte Carlo simulation study, in Conference Rec. of 1998 IEEE Nuclear Science Symposium \& Medical Imaging Conference (8-14 November, Toronto, Ontario, Canada), 1 (1999) 577. IEEE Trans. Nucl. Sci., accepted. 